\title{
A New Beginning for the Journal of Minimally Invasive Surgery for the International Recognition and Contribution of Scientific Development of Minimally Invasive Surgery
}

\author{
Suk Hwan Lee, M.D., Ph.D. \\ Department of Surgery, Kyung Hee University Hospital at Gangdong, Seoul, Korea
}

Corresponding author

Suk Hwan Lee

Department of Surgery, Kyung Hee Univesity College of Medicine, 892 Dongnam-ro, Gangdong-gu, Seoul 05278, Korea

Tel: +82-2-440-6134, Fax: +82-2-440-6073, E-mail: leeshdr@khu.ac.kr

This is an Open Access article distributed under the terms of the Creative Commons Attribution Non-Commercial License (http:// creativecommons.org/licenses/by-nc/4.0/) which permits unrestricted non-commercial use, distribution, and reproduction in any

Copyright $(92017$ The Journal of Minimally medium, provided the original work is properly cited.

Invasive Surgery. All rights reserved.

This year marks a new beginning for the Journal of Minimally Invasive Surgery (J Minim Invasive Surg) because we have changed our publication language policy from Korean to English. The Journal of Minimally Invasive Surgery is the official journal of The Korean Society of Endoscopic and Laparoscopic Surgeons (KSELS), which represents researchers in all fields using minimally invasive approaches such as endoscopy or laparoscopy for the purpose of diagnosis or treatment. The aims of this journal are to contribute scientific advances and studies of surgery exploiting endoscopy or laparoscopy and to facilitate the exchange of information and development of research among mutual members.

After the first successful implementation of laparoscopic cholecystectomy in Korea in early 1990, KSELS was organized in April of 1996 by 109 founding members for the purpose of distributing literature pertaining to endoscopic and laparoscopic operations and facilitating future development of various laparoscopic surgeries. The journal was launched as Daehan Naesigyeong Bokganggyeong Hakhoeji, which translates to the Journal of The Korean Society of Endoscopic and Laparoscopic Surgeons, as a biannual publication in May of 1998 (ISSN 1738-7884 from 2005). We initially did not organize editorial members for our journal because the society itself was so small and primitive in terms of organization, and most of the laparoscopic surgeries conducted at that time were cholecystectomies or appendectomies. Rather, the Secretary General of the society was in charge of publication of the journal. There was also no peer-review processes in place when publication first started. The Korean Association of Medical Journal Editors (KAMJE) was established in 1996 to improve the quality of medical journal publications in $\mathrm{Ko}^{-}$ rea. Under the guidance of the KAMJE, the first editor-inchief (Prof. Jun-Gi Kim) was appointed in 2006. Prof. JunGi Kim served for two years as editor-in-chief of Daehan Naesigyeong Bokganggyeong Hakhoeji. During this period, our journal began a peer-review process for submitted papers. Professor Hyung-Ho Kim was appointed as the second editorin-chief in April of 2008. Prof. Hyung-Ho Kim made many contributions that greatly improved the quality of our journal. For example, he developed an on-line submission system and changed the name to the Journal of Minimally Invasive Surgery in 2011 (ISSN 2234-778X) to increase its international recognition. From 2012 (volume 15, No. 1), we started to publish our journal quarterly (every $15^{\text {th }}$ of March, June, September and December). Some predictable difficulties were encountered with this change, such as a shortage of papers 


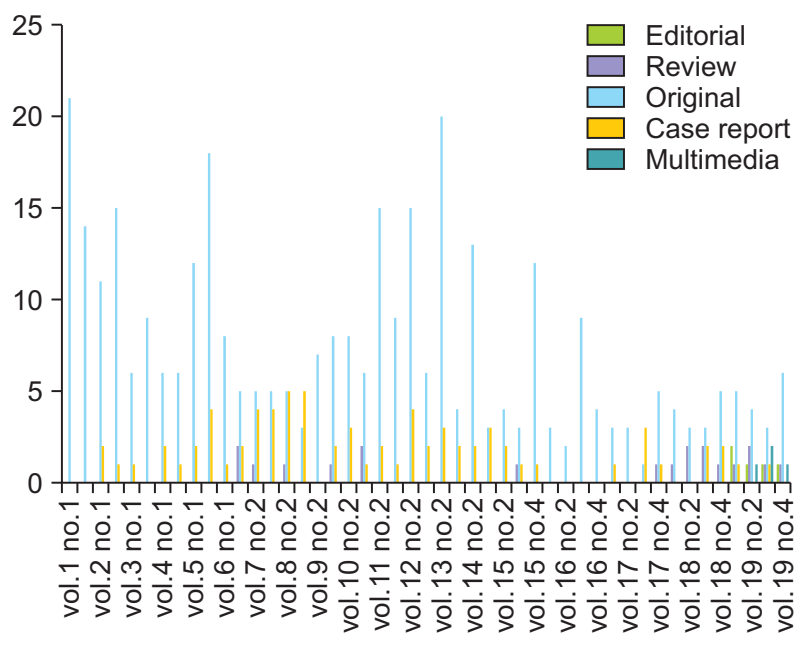

Fig. 1. Graphic demonstration of number of articles on Journal of Minimally Invasive Surgery from Vol. 1, No. 1 (1998) to Vol.19, №.4 (2016).

submitted for the journal (Fig. 1). This phenomenon was contrasted with increasing submission of internationally known biomedical journals from Korea. However, entry of the MeSH terms "laparoscopy" and "Korea" into PubMed searches revealed a clear increase in Korean submissions to international journals from 2009 (Fig. 2). By 2015, more than 300 papers were published internationally; therefore, it became necessary for our journal to change its publication policies to improve quality and ensure our survival as a biomedical publication.

I (Suk-Hwan Lee) was appointed as the third editor-inchief of the journal in April of 2014. Since then, we have further improved the quality of our journal. Specifically, we changed the overall format of our journal. We also started to publish review articles regularly from Vol. 17 No. 4 (2014), and we began sending e-mails to our subscribers showing the table of contents of upcoming issues in 2015 (vol. 18, No. 1). We have also changed the journal to be more reader friendly and full color (Vol. 18, No. 1, 2015). Finally, we decided to publish multimedia articles to highlight and enhance the distribution of laparoscopic and endoscopic procedures and knowledge.

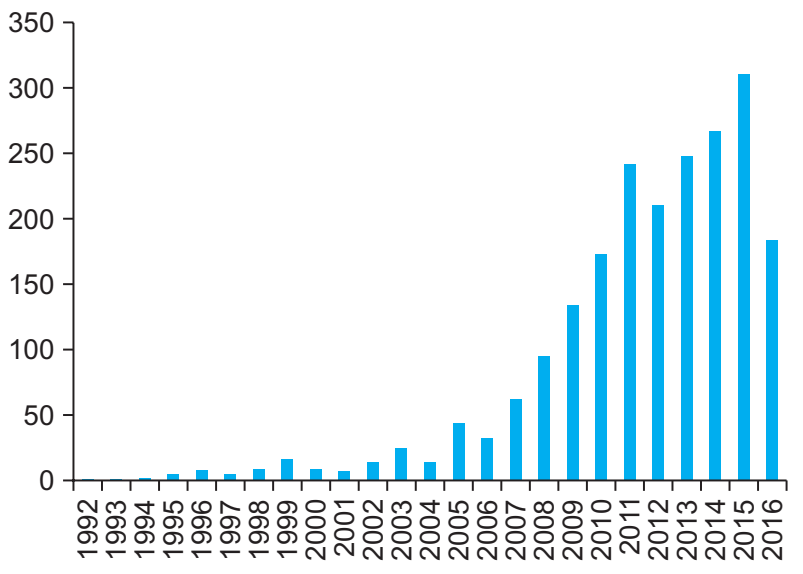

Fig. 2. Number of Korean literatures submitted international biomedical journals from 1992 to 2016. Pubmed search, MeSH keywords "laparoscopy" and "Korea".

The first multimedia article was published last year (Vol. 19, No. 2), and it is anticipated that multimedia articles will become the primary medium by which we propagate minimally invasive surgical techniques and know-how to readers.

Because English is the main global language for scientific communication, we changed the language policy of the journal to English from this issue. With these continuing efforts to improve the quality of our journal, we believe it will be indexed in the United States National Library of Medicine database in the near future.

Our society has gained international recognition because of our members' efforts toward continual development of minimally invasive surgical techniques, such as robotic surgery and single port laparoscopic surgeries. It is now time to improve our journal's recognition to those of our members.

I hope all members of KSELS advise editorial members of any ideas they have to improve the quality of our journal and submit their valuable research for academic development of minimally invasive surgical fields. 